James P. Harrison

Der lange Marsch zur Macht.

Die Geschichte der kommunistischen Partei Chinas von ihrer Gründung bis zum Tode von Mao Tse-tung.

Belser Verlag, Stuttgart, Zürich, 1978, 880 S., DM 49,-

Das Werk des New Yorker Historikers Harrison erschien in englischer Sprache im Jahre 1972 (,,The Long March to Power”, Praeger Publishers). Es handelt sich um eine chinesische und sow jetische Quellen einbeziehende, freilich ausweislich des Vorworts hauptsächlich auf Sekundärquellen gestützte, detaillierte Schilderung des Weges der Kommunistischen Partei Chinas von der Gründung im Juli 1921 bis zum Beginn der siebziger Jahre: Wenn der Untertitel mehr verheißt, so ist das nur formal korrekt, denn die Entwicklung von 1970 bis 1976 wird nur auf wenigen Seiten skizziert, die für die deutsche Ausgabe angefügt worden sind. Harrison widmet der Vorkriegsgeschichte mehr als zwei Drittel des Bandes. Die Bedeutung des Nationalismus und der Idee der Massenlinie für die Entwicklung der KPCh sowie die Fähigkeit insbesondere des jüngeren Mao Tse-tung, integrierende Mittelkurse zu steuern, werden immer wieder deutlich gemacht. Faktenreichtum, klare Gliederung, auch Karten, Statistiken, Úbersichten ließen bereits die amerikanische Ausgabe zum unentbehrlichen Nachschlagewerk werden. Das Erscheinen der sehr gut lesbaren, mit zahlreichen Fotografien versehenen deutschen Ubersetzung zu einem erträglichen Preis verdient Beifall.

Philip Kunig

\title{
Hans Schemann/Luiza Schemann-Dias
}

Dicionário idiomático português-alemão/Portugiesisch-deutsche Idiomatik Max Hueber Verlag, Ismaning/München 1978, 912 Seiten, DM 84,-.

Nicht nur der absehbare Eintritt Portugals in die Europäische Gemeinschaft, sondern auch die rapide Expansion der brasilianisch-deutschen Beziehungen läßt das Bedürfnis nach Hilfsmitteln für die zunehmend notwendigen Sprachstudien entsprechend steigen. Eine ganz wesentliche Bereicherung des bislang eher dürftig ausgestatteten deutschen Marktes die einzige Alternative etwa zum nachgerade antiquierten ,,Langenscheidt“ im Bereich der Taschenwörterbücher stammt vom Verlag Enzyklopädie Leipzig - ist das neue idiomatische Wörterbuch von Schemann/Schemann-Dias. Der vorliegende portugiesisch-deutsche Band, dem bald auch ein deutsch-portugiesischer Parallel-Band folgen soll, enthält in alphabetischer Anordnung etwa 8000 idiomatische Wendungen. Jedem portugiesischen Idiom steht die deutsche Entsprechung gegenüber, nach Möglichkeit ebenfalls in idiomatischer Form. Der Praktikabilität des Bandes sehr förderlich ist jeweils die Angabe der Stilebene, wobei die Autoren deren acht unterscheiden: Gehoben, literarisch - gehoben/formelhaft, mit Tendenz zu Kitsch - Amtsstil - Briefstil - terminus technicus - umgangssprachlich, ,, normal“ - familiär, ,,sorglos" - vulgär, grob, scharf -. Ebenso dankbar wird jeder Benutzer sein für die zumeist angefügten Beispielsätze, die die jeweilige Anwendung im authentischen Kontext illustrieren. Die Unterschiede im portugiesischen und brasilianischen Sprachgebrauch sind dabei selbstverständlich hervorgehoben.

Dem eigentlichen idiomatischenTeil vorausgeschickt ist eine breit angelegte zweisprachige „,Einführung“" (S. VII-XLVII). Dabei mag dahinstehen, ob die Autoren gut beraten waren, den bei einem solchen Werk doppelt nötigen praktischen Gebrauchshinweisen mit 8 Seiten nur relativ wenig Raum zu widmen. Den Löwenanteil verschlingt ein Essay über ,,Die methodologischen Voraussetzungen der portugiesisch-deutschen Idiomatik", verfaßt mit be- 
eindruckender fachwissenschaftlicher Terminologie, für den Praktiker jedoch so gut wie unzugänglich. Auch finden sich unter den 8000 Wendungen mitunter manche, über deren Aufnahme zu streiten wäre, weil sich ihr Sinn über jedes normale Wörterbuch erschließt, z. B. „fazer bicha“"(S. 63), ,,ser uma autêntica nulidade“ (S. 493), ,,ganhar o seu pão com o suor de seu rosto“ (S. 545), ,,cair de sono" (S. 747).

Die Qualität des Werkes vermögen indes solche und andere ,,bagateles“ nicht entscheidend zu mindern. Der Verlag hat einen Band vorgelegt, der zwar vornehmlich in die Hand des Fortgeschrittenen gehört, der aber auch dem Anfänger bereits wertvolle Dienste leisten kann. Und dies zu einem keineswegs prohibitiven Preis.

Karl Hernekamp 